\title{
Global solutions and finite time blow up for damped semilinear wave equations is $^{\text {s }}$
}

\author{
Filippo Gazzola*, Marco Squassina \\ Dipartimento di Matematica “F. Brioschi”, Politecnico di Milano, Via Bonardi 9, 20133 Milano, Italy
}

Received 13 January 2005; received in revised form 24 February 2005; accepted 28 February 2005

Available online 13 June 2005

\begin{abstract}
A class of damped wave equations with superlinear source term is considered. It is shown that every global solution is uniformly bounded in the natural phase space. Global existence of solutions with initial data in the potential well is obtained. Finally, not only finite time blow up for solutions starting in the unstable set is proved, but also high energy initial data for which the solution blows up are constructed.
\end{abstract}

(C) 2006 L'Association Publications de l'Institut Henri Poincaré. Published by Elsevier B.V. All rights reserved

MSC: 35L 70; 35B 40

Keywords: Damped wave equations; Stable and unstable set; Global solutions; Blowing up solutions; Convergence to equilibria; Nehari manifold; Mountain pass level

\section{Introduction}

We study the behavior of local solutions of the following superlinear hyperbolic equation with (possibly strong) linear damping

$$
\begin{cases}u_{t t}-\Delta u-\omega \Delta u_{t}+\mu u_{t}=|u|^{p-2} u & \text { in }[0, T] \times \Omega, \\ u(0, x)=u_{0}(x) & \text { in } \Omega, \\ u_{t}(0, x)=u_{1}(x) & \text { in } \Omega, \\ u(t, x)=0 & \text { on }[0, T] \times \partial \Omega\end{cases}
$$

where $\Omega$ is an open bounded Lipschitz subset of $\mathbb{R}^{n}(n \geqslant 1), T>0$,

\footnotetext{
th The first author was partially supported by the Italian MIUR Project "Calcolo delle Variazioni" while the second author was partially supported by the Italian MIUR Project "Metodi Variazionali e Topologici nello Studio dei Fenomeni Nonlineari” and by the INdAM.

* Corresponding author.

E-mail addresses: gazzola@ mate.polimi.it (F. Gazzola), squassina@mate.polimi.it (M. Squassina).
} 


$$
\begin{aligned}
& u_{0} \in H_{0}^{1}(\Omega), \quad u_{1} \in L^{2}(\Omega), \\
& \omega \geqslant 0, \quad \mu>-\omega \lambda_{1},
\end{aligned}
$$

$\lambda_{1}$ being the first eigenvalue of the operator $-\Delta$ under homogeneous Dirichlet boundary conditions, and

$$
2<p \leqslant\left\{\begin{array}{ll}
\frac{2 n}{n-2} & \text { for } \omega>0 \\
\frac{2 n-2}{n-2} & \text { for } \omega=0
\end{array} \text { if } n \geqslant 3, \quad 2<p<\infty \quad \text { if } n=1,2 .\right.
$$

We study the behavior of solutions to $(1.1)$ in the phase space $H_{0}^{1}(\Omega)$. Since stationary solutions play a crucial role in the description of the evolution of (1.1), several tools from critical point theory turn out to be quite useful for our purposes. In particular, we consider the mountain pass energy level d (see e.g. [1]), the Nehari manifold $\mathscr{N}$ (see [20]) of the stationary problem associated to (1.1) and the two unbounded sets $\mathscr{N}_{+}$(inside $\mathscr{N}$ ) and $\mathscr{N}_{-}$ (outside $\mathscr{N}$ ). All these tools are defined in detail in Section 2. A first attempt to tackle (1.1) with these tools was made by Sattinger [27] (see also [24,28]) who developed the so-called potential well theory in order to study the problem with no damping (that is $\omega=\mu=0$ ). Subsequently, equations with damping terms have been considered by many authors. For equations with (possibly nonlinear) weak damping we refer to [9,13,14,18,25,29]. Much less is known for equations with strong damping; see the seminal paper by Levine [17] (and also [21,22]) but still many problems remain unsolved. It is our purpose to shed some further light on damped wave equations of the kind of (1.1) in both the cases of weak $(\omega=0)$ and strong $(\omega>0)$ damping. To this end, as recently done by the first author in $[7,8]$ for parabolic equations, we will exploit further the properties of the Nehari manifold. In particular, this will enable us to obtain blow up results in correspondence of initial data $\left(u_{0}, u_{1}\right)$ having arbitrarily large initial energy. As far as we are aware, this is the first blow up result for (1.1) with $E(0)>d$ (initial energy above the mountain pass level). However we mention that, by exploiting a completely different method, the existence of solutions with arbitrarily high initial energy has been also obtained in [19] for weakly damped wave equations on the whole $\mathbb{R}^{n}$.

Let us explain in some detail which are our main results. We first make clear for which exponents $p$ problem (1.1) is (locally) well posed. We restricted our attention to the superlinear case $p>2$ since the sublinear case $p \in(1,2]$ is well established (see Remark 3.10). When $\omega=0$ and $\mu>0$, it is proved in [11] that (1.1), (1.2) admits a unique local weak solution for any $p>2$ if $n=1,2$ and for $2<p \leqslant \frac{2 n-2}{n-2}$ if $n \geqslant 3$; note that $\frac{2 n-2}{n-2}$ is the critical exponent $r$ for the trace embedding $H^{1}(\Omega) \subset L^{r}(\partial \Omega)$. We wish to stress that, leaving aside the well posedness of (1.1), the constraint $p \leqslant \frac{2 n-2}{n-2}$ for $\omega=0$ and initial data (1.2) is up to now unavoidable for the energy identity to make sense, i.e. it is not known if formula (4.13) holds for $p>\frac{2 n-2}{n-2}$ : we refer to [2] for further comments. In Theorem 3.1 we show that in presence of a strong damping $(\omega>0)$ this upper bound for $p$ can be enlarged to $p \leqslant \frac{2 n}{n-2}$, which is the "natural" constraint since $2^{*}=\frac{2 n}{n-2}$ is the critical Sobolev exponent $q$ for the embedding $H_{0}^{1}(\Omega) \hookrightarrow L^{q}(\Omega)$. Our result restates [22, Theorem 1] for a wider class of initial data but for a smaller range of exponents $p$. When dealing with critical point theory, the correct phase space for the solutions of (1.1) is necessarily $H_{0}^{1}(\Omega)$ and, therefore, the natural regularity for the initial data is precisely that of (1.2).

Cazenave [4] proved boundedness of global solutions to (1.1) for $\omega=\mu=0$ while Esquivel-Avila [5] recovered the same result for $\omega=0$ and $\mu>0$ and showed that this property may fail in presence of a nonlinear dissipation term (cf. [6, Theorem 3.4]). In Theorems 3.4 and 3.6, by exploiting an argument different from the one devised in [4,5], we prove that any global solution of (1.1) is bounded whenever $\omega$ and $\mu$ fulfill (1.3). The proof relies on a delicate analysis of the behavior of several norms of the solution as $t \rightarrow \infty$. Moreover, we obtain convergence up to a subsequence of solutions of (1.1) towards a steady-state $\phi$. Since in general the source nonlinearity $\left\{u \mapsto|u|^{p-2} u\right\}$ in (1.1) is not an analytic function, counterexamples of Jendoubi-Poláčik [15] show that we cannot expect that all global solutions $u=u(t)$ stabilize, that is

$$
\lim _{t \rightarrow \infty}\left\|u_{t}(t)\right\|_{2}+\|\nabla u(t)-\phi\|_{2}=0 .
$$

Only under more restrictive assumptions on $n$ and $p$, one may guarantee that (1.5) indeed occurs, see Remark 3.7. 
Once boundedness of global solutions is established, one is interested to find out for which initial data (1.2) problem (1.1) does have a global solution. For the undamped equation $(\omega=\mu=0)$ Sattinger [27] showed that local solutions of (1.1) are in fact global whenever $E(0)<d$ and $u_{0} \in \mathscr{N}_{+}$. This statement may be improved in presence of dissipation; for the weakly damped equation $(\omega=0, \mu>0)$ Ikehata and Suzuki [14] prove that under the same assumptions on the initial data, not only the solution is global but it also converges to the equilibrium $\phi \equiv 0$ as $t \rightarrow \infty$. In Theorem 3.8 we extend this result to the case $\omega>0$. Our result improves [22, Theorem 3] where $E(0) \leqslant d / 2^{2 /(p-2)}$ and only the case $\mu=0$ is considered.

Not all local solutions of (1.1) are global in time. Blow up in finite time is usually obtained for low initial energy $E(0)$ and for $u_{0} \in \mathscr{N}_{-}$. For the undamped equation $(\omega=\mu=0)$ Tsutsumi [28] showed that local solutions of (1.1) cannot be continued to the whole $[0, \infty)$ provided that $u_{0} \in \mathscr{N}_{-}$and $E(0)<d$. For equations with weak damping $(\omega=0$ and $\mu>0)$, Levine and Serrin [18] proved nonexistence of global solutions when $E(0)<0$, a condition which automatically implies that $u_{0} \in \mathscr{N}_{-}$. Subsequently, Ikehata and Suzuki [13,14] proved the same result when $u_{0} \in \mathscr{N}_{-}$and $E(0)<d-\varepsilon$ for a suitable $\varepsilon \in(0, d)$ depending on the damping coefficient $\mu$. Finally, Pucci and Serrin [25] successfully handled the case when $E(0)<d$ and Vitillaro [29] showed that also for $E(0)=d$ the solution blows up in finite time. When $\omega>0$ and $\mu=0$, Ono [22, Theorem 7] shows that the solution of (1.1) blows up in finite time if $E(0)<0$. For the same problem, Ohta [21] improves this result by allowing $E(0)<d$, provided that $u_{0} \in \mathscr{N}_{-}$. In Theorem 3.11 , by refining and simplifying the concavity method introduced by Levine $[16,17]$, we extend this result to the case where $\mu \neq 0$ and $E(0) \leqslant d$. Last but not least, in Theorems 3.12 and 3.13 we show the finite time blow up of some solutions of (1.1) whose initial data have arbitrarily high initial energy. The proof is inspired by previous work in [8] and uses the weak antidissipativity of the flow in $\mathscr{N}_{-}$.

This paper is organized as follows.

- in Section 2 we recall some preliminary tools and definitions;

- in Section 3 we present the main results of the paper and we list some open problems;

- from Sections 4 to 10 we provide the proofs of the results. We point out that the proofs are not in the same order as the statements.

\section{Setup and notations}

We denote by $\|\cdot\|_{q}$ the $L^{q}(\Omega)$ norm for $1 \leqslant q \leqslant \infty$ and by $\|\nabla \cdot\|_{2}$ the Dirichlet norm in $H_{0}^{1}(\Omega)$. Moreover, for later use we denote by $\langle\cdot, \cdot\rangle$ the duality pairing between $H^{-1}(\Omega)$ and $H_{0}^{1}(\Omega)$. When $\omega>0$ (resp. $\omega=0$ ) for all $v, w \in H_{0}^{1}(\Omega)$ (resp. for all $v, w \in L^{2}(\Omega)$ ), we put

$$
(v, w)_{*}=\omega \int_{\Omega} \nabla v \cdot \nabla w+\mu \int_{\Omega} v w, \quad\|v\|_{*}=(v, v)_{*}^{1 / 2} ;
$$

by (1.3), $\|\cdot\|_{*}$ is an equivalent norm over $H_{0}^{1}(\Omega)\left(\right.$ resp. $\left.L^{2}(\Omega)\right)$.

By (1.4), we may consider the $C^{1}$ functionals $I, J: H_{0}^{1}(\Omega) \rightarrow \mathbb{R}$ defined by

$$
I(u)=\|\nabla u\|_{2}^{2}-\|u\|_{p}^{p} \quad \text { and } \quad J(u)=\frac{1}{2}\|\nabla u\|_{2}^{2}-\frac{1}{p}\|u\|_{p}^{p} .
$$

The mountain pass value of $J$ (also known as potential well depth) is defined as

$$
d=\inf _{u \in H_{0}^{1}(\Omega) \backslash\{0\}} \max _{\lambda \geqslant 0} J(\lambda u) .
$$


Consider the best Sobolev constant for the embedding $H_{0}^{1}(\Omega) \hookrightarrow L^{p}(\Omega)$,

$$
S_{p}=\inf _{u \in H_{0}^{1}(\Omega) \backslash\{0\}} \frac{\|\nabla u\|_{2}^{2}}{\|u\|_{p}^{2}} .
$$

If $(n-2) p<2 n$, the embedding is compact and the infimum in (2.2) (and in (2.1)) is attained. In such case (see, e.g. [24, Section 3]), any mountain pass solution $u$ of the stationary problem is a minimizer for (2.2) (i.e. it satisfies $\|\nabla u\|_{2}^{2}=S_{p}\|u\|_{p}^{2}$ ) and $S_{p}$ is related to its energy

$$
d=\frac{p-2}{2 p} S_{p}^{p /(p-2)} .
$$

All nontrivial stationary solutions belong to the so-called Nehari manifold (see [20] and also [31]) defined by

$$
\mathscr{N}=\left\{u \in H_{0}^{1}(\Omega) \backslash\{0\}: I(u)=0\right\} .
$$

It is easy to show that each half line starting from the origin of $H_{0}^{1}(\Omega)$ intersects exactly once the manifold $\mathscr{N}$ and that $\mathscr{N}$ separates the two unbounded sets

$$
\mathscr{N}_{+}=\left\{u \in H_{0}^{1}(\Omega): I(u)>0\right\} \cup\{0\} \quad \text { and } \quad \mathscr{N}_{-}=\left\{u \in H_{0}^{1}(\Omega): I(u)<0\right\} .
$$

We also consider the (closed) sublevels of $J$

$$
J^{a}=\left\{u \in H_{0}^{1}(\Omega): J(u) \leqslant a\right\} \quad(a \in \mathbb{R})
$$

and we introduce the stable set $\mathscr{W}$ and the unstable set $\mathscr{U}$ defined by

$$
\mathscr{W}=J^{d} \cap \mathscr{N}_{+} \quad \text { and } \quad \mathscr{U}=J^{d} \cap \mathscr{N}_{-} .
$$

It is readily seen (see [31, Theorem 4.2]) that the mountain pass level $d$ defined in (2.1) may also be characterized as

$$
d=\inf _{u \in \mathscr{N}} J(u) .
$$

This alternative characterization of $d$ shows that

$$
\beta=\operatorname{dist}(0, \mathscr{N})=\inf _{u \in \mathscr{N}}\|\nabla u\|_{2}=\sqrt{\frac{2 d p}{p-2}}>0
$$

and that, for every $a>d$, we have

$$
\mathscr{N}_{a}=\mathscr{N} \cap J^{a} \equiv\left\{u \in \mathscr{N}:\|\nabla u\|_{2} \leqslant \sqrt{\frac{2 a p}{p-2}}\right\} \neq \emptyset .
$$

Therefore, for every $a>d$, we may define

$$
\Lambda_{a}=\sup \left\{\|u\|_{2}: u \in \mathscr{N}_{a}\right\} .
$$

By Poincaré inequality, we have $\Lambda_{a}<\infty$ for every $a>d$. We introduce the sets

$$
\begin{aligned}
& \mathscr{S}=\left\{\phi \in H_{0}^{1}(\Omega): \phi \text { is a stationary solution of }(1.1)\right\}, \\
& \mathscr{S}_{\ell}=\{\phi \in \mathscr{S}: J(\phi)=\ell\} \quad\left(\ell \in \mathbb{R}_{+}\right) .
\end{aligned}
$$

Finally, we consider the energy functional $\mathscr{E}: H_{0}^{1}(\Omega) \times L^{2}(\Omega) \rightarrow \mathbb{R}$ defined by

$$
\mathscr{E}(v, w)=J(v)+\frac{1}{2}\|w\|_{2}^{2}
$$

and the Lyapunov function $E(t)=\mathscr{E}\left(u(t), u_{t}(t)\right)$, defined for any solution $u(t)$ to (1.1). 


\section{The main results}

By solution of (1.1), (1.2) over [0,T] we mean a function

$$
u \in C^{0}\left([0, T], H_{0}^{1}(\Omega)\right) \cap C^{1}\left([0, T], L^{2}(\Omega)\right) \cap C^{2}\left([0, T], H^{-1}(\Omega)\right),
$$

with $u_{t} \in L^{2}\left([0, T], H_{0}^{1}(\Omega)\right)$ whenever $\omega>0$, such that $u(0)=u_{0}, u_{t}(0)=u_{1}$ and

$$
\left\langle u_{t t}(t), \eta\right\rangle+\int_{\Omega} \nabla u(t) \cdot \nabla \eta+\omega \int_{\Omega} \nabla u_{t}(t) \cdot \nabla \eta+\mu \int_{\Omega} u_{t}(t) \eta=\int_{\Omega}|u(t)|^{p-2} u(t) \eta
$$

for all $\eta \in H_{0}^{1}(\Omega)$ and a.e. $t \in[0, T]$.

We first establish local existence and uniqueness for solutions of (1.1), (1.2).

Theorem 3.1. Assume that (1.3) and (1.4) hold. Then there exist $T>0$ and a unique solution of (1.1), (1.2) over $[0, T]$. Moreover, if

$$
T_{\max }=\sup \{T>0: u=u(t) \text { exists on }[0, T]\}<\infty
$$

then

$$
\lim _{t \rightarrow T_{\max }}\|u(t)\|_{q}=\infty \quad \text { for all } q \geqslant 1 \text { such that } q>\frac{n(p-2)}{2}
$$

if $n \geqslant 3$ and $p=2^{*}$ (so that $\omega>0$ ), then (3.1) also holds for $q=\frac{n(p-2)}{2}=2^{*}$.

Definition 3.2. If $T_{\max }<\infty$, we say that the solution of (1.1), (1.2) blows up and that $T_{\max }$ is the blow up time. If $T_{\max }=\infty$, we say that the solution is global. The property of continuing (in time) a bounded solution will be referred throughout the paper as the Continuation Principle.

Remark 3.3. As it should be expected, from the proof of Theorem 3.1 it follows that, for fixed initial data, we have $T_{\max } \rightarrow \infty$ as $\omega \rightarrow \infty$, that is to say, the more the equation gets damped, the larger becomes the life span of the solution.

Next, we prove the boundedness of global solutions $u$, namely

$$
u \in L^{\infty}\left(\mathbb{R}_{+}, H_{0}^{1}(\Omega)\right) \cap W^{1, \infty}\left(\mathbb{R}_{+}, L^{2}(\Omega)\right) .
$$

In the strongly damped case we have the following

Theorem 3.4. Assume that $\omega>0$ and that (1.3) and (1.4) hold. Then, every global solution $u(t)$ to (1.1), (1.2) satisfies (3.2). Moreover, if $n=1,2$ or if

$$
n \geqslant 3 \text { and } 2<p<2^{*}
$$

then there exists $\ell \in \mathbb{R}_{+}$such that $\mathscr{S}_{\ell} \neq \emptyset$,

$$
\lim _{t \rightarrow \infty} E(t)=\ell, \quad \lim _{t \rightarrow \infty} \operatorname{dist}_{H_{0}^{1}}\left(u(t), \mathscr{S}_{\ell}\right)=0 \quad \text { and } \quad \lim _{t \rightarrow \infty}\left\|u_{t}(t)\right\|_{2}=0,
$$

and there exist $\left\{t_{j}\right\} \subset \mathbb{R}_{+}$with $t_{j} \rightarrow \infty$ and $\phi \in \mathscr{S}_{\ell}$ such that

$$
\lim _{j \rightarrow \infty}\left\|\nabla u\left(t_{j}\right)-\nabla \phi\right\|_{2}=0 .
$$


Remark 3.5. Assume that $n \geqslant 3$ and that $\Omega$ is star shaped. Then, in the limiting case $p=2^{*}$, the well known Pohǒzaev identity (see e.g. [31, Theorem B.1]) combined with the unique continuation property for elliptic equations yields $\mathscr{S}=\{0\}$. Then, arguing as in the proof of Theorem 3.4, it is possible to show that from every global solution $u=u(t)$ we may extract a subsequence $\left\{u\left(t_{j}\right)\right\}$ such that $u\left(t_{j}\right) \rightarrow 0$ weakly in $H_{0}^{1}(\Omega)$, while the strong convergence $u\left(t_{j}\right) \rightarrow 0$ seems to be out of reach.

In order to prove the boundedness of global solutions (cf. (3.2)) we make use of a delicate analysis of all the terms involved in (1.1), see Section 7.1. The corresponding statement for the weakly damped case $(\omega=0)$ has recently been obtained by Esquivel-Avila [5]. Based on the just mentioned delicate analysis, in Section 7.2 we give a different proof of the following

Theorem 3.6 [5]. Assume that $\omega=0$ and that

$$
2<p \leqslant\left\{\begin{array}{ll}
\frac{2 n-2}{n-2} & \text { for } n \geqslant 3, \\
6 & \text { for } n=2,
\end{array} \quad 2<p<\infty \quad \text { for } n=1 .\right.
$$

Then, every global solution $u(t)$ to (1.1), (1.2) satisfies (3.2). Moreover, if $n=1,2$ or if

$$
2<p<\frac{2 n-2}{n-2} \text { for } n \geqslant 3,
$$

then there exists $\ell \in \mathbb{R}_{+}$such that $\mathscr{S}_{\ell} \neq \emptyset$, (3.4) holds and there exist $\left\{t_{j}\right\} \subset \mathbb{R}_{+}$with $t_{j} \rightarrow \infty$ and $\phi \in \mathscr{S}_{\ell}$ such that (3.5) holds.

Remark 3.7. By combining the boundedness of global solutions that we obtained in Theorems 3.4 and 3.6 with some well known convergence results one can prove stabilization of the whole flow. In one space dimension, for any $p>2$, under assumption (1.3) one obtains (1.5) for some equilibrium $\phi$, as a consequence of [10, Theorem 2.4]; follow step by step the arguments of Section 5.4 therein, with the only difference that the orbit precompactness is due to (3.5) and not as a byproduct of the existence of the global attractor. In two space dimensions, the situation is different for weak and strong damping; if $\omega=0$ one has (1.5) for $p=4,6$ (see [12, Theorem 1.2]), whereas if $\omega>0$ one has (1.5) for any even integer $p \geqslant 4$ (see [12, Theorem 4.4.1 and Example 4.4.1]).

Let us turn to the global existence of solutions starting with suitable initial data.

Theorem 3.8. Assume that (1.3) and (1.4) hold and let $u$ be the unique local solution to (1.1), (1.2). In addition, assume that there exists $\bar{t} \in\left[0, T_{\max }\right)$ such that

$$
u(\bar{t}) \in \mathscr{W} \quad \text { and } \quad \mathscr{E}\left(u(\bar{t}), u_{t}(\bar{t})\right) \leqslant d .
$$

Then $T_{\max }=\infty$ and, for every $t>\bar{t}$,

$$
\|\nabla u(t)\|_{2}^{2}+\left\|u_{t}(t)\right\|_{2}^{2} \leqslant \frac{\Theta(\omega, \mu)}{t}
$$

where

$$
\Theta(\omega, \mu)= \begin{cases}C_{\mu}\left(1+\frac{1}{\omega}+\omega\right) & \text { for } \omega>0, \\ C\left(1+\frac{1}{\mu}+\mu\right) & \text { for } \omega=0\end{cases}
$$

and $C$ is independent of $\mu$, whereas $C_{\mu}$ only depends on $\mu$.

Remark 3.9. Let $\omega>0$ and $\mu=0$. Although inequality (3.9) gives only a one-sided control, since $\Theta(\omega, 0) \rightarrow \infty$ both for $\omega \rightarrow 0$ and $\omega \rightarrow \infty$, the best dissipation rate for the energy norm (with respect to the damping coefficient $\omega$ ) seems to be achieved at the minimum point of $\Theta(\omega, 0)$, which occurs at $\omega=1$. Physically, as $\omega \rightarrow 0$ the 
dissipation gets lost, whereas for $\omega \rightarrow \infty$ the system tends to freeze since $\omega$ acts only on the velocity $u_{t}$. A similar phenomenon has been observed for a (different) class of strongly damped wave equations in [23, Remarks 4 and 5], in discussing the size of the universal attractor as $\omega$ varies.

Remark 3.10. In the case $p \in(1,2]$, we have $T_{\max }=\infty$ for arbitrary choices of the initial data (1.2). Indeed, for any fixed $T>0$, define the functional $\Psi:[0, T] \rightarrow \mathbb{R}^{+}$,

$$
\left.\Psi(t)=\frac{1}{2}\|\nabla u(t)\|_{2}^{2}+\frac{1}{2} \| u_{t}(t)\right)\left\|_{2}^{2}+\frac{1}{p}\right\| u(t) \|_{p}^{p} .
$$

Notice that, for every $t \in[0, T]$, there holds

$$
\Psi^{\prime}(t) \leqslant-\left\|u_{t}(t)\right\|_{*}^{2}+2 \int_{\Omega}|u(t)|^{p-1}\left|u_{t}(t)\right| .
$$

Since $2 n(p-1) /(n+2) \leqslant p$, by Sobolev and Young inequalities, we have

$$
2 \int_{\Omega}|u|^{p-1}\left|u_{t}\right| \leqslant c\left\|\nabla u_{t}\right\|_{2}\|u\|_{p}^{p-1} \leqslant\left\|u_{t}\right\|_{*}^{2}+c \Psi^{2(p-1) / p} .
$$

Here and below $c$ denotes a positive constant. Therefore, we get

$$
\Psi^{\prime}(t) \leqslant c \Psi^{2(p-1) / p}(t) \quad \text { for every } t \in[0, T] .
$$

Since $p \in(1,2]$, we have $2(p-1) / p \in(0,1]$ and

$$
\left.\|\nabla u(t)\|_{2}^{2}+\| u_{t}(t)\right) \|_{2}^{2} \leqslant 2 \Psi(t) \leqslant \begin{cases}c(T+c)^{p /(2-p)} & \text { for } p<2 \\ c \mathrm{e}^{c T} & \text { for } p=2 .\end{cases}
$$

By the continuation principle, the solution has to be globally defined.

We come to a blow up result for solutions starting in the unstable set.

Theorem 3.11. Assume that (1.3) and (1.4) hold and let $u$ be the unique local solution to (1.1), (1.2). Then $T_{\max }<\infty$ if and only if there exists $\bar{t} \in\left[0, T_{\max }\right)$ such that

$$
u(\bar{t}) \in \mathscr{U} \quad \text { and } \quad \mathscr{E}\left(u(\bar{t}), u_{t}(\bar{t})\right) \leqslant d .
$$

Theorem 3.11 is already known for weakly damped equations $(\omega=0)$, see [29]. In Section 6 we give a general proof of this statement under the sole assumption (1.3). As a byproduct of our proof it is clear that $T_{\max }<\infty$ if and only if $E(t) \rightarrow-\infty$ as $t \rightarrow T_{\max }$. In particular, the blow up has a full characterization in terms of (negative) energy blow up.

In the weakly damped case we state the blow up of solutions to (1.1) with suitable initial data having energy larger than the mountain pass level $d$.

Theorem 3.12. Assume that $\omega=0$ and $\mu \geqslant 0$ and that (1.4) holds. In addition, assume that $\left(u_{0}, u_{1}\right) \in \mathscr{N}_{-} \times L^{2}(\Omega)$ are such that

$$
\mathscr{E}\left(u_{0}, u_{1}\right)>d, \quad\left\|u_{0}\right\|_{2} \geqslant \Lambda_{\mathscr{E}\left(u_{0}, u_{1}\right)}, \quad \int_{\Omega} u_{0} u_{1} \geqslant 0 .
$$

Then $T_{\max }<\infty$ for the corresponding solution $u$ of (1.1), (1.2).

As a consequence of Theorem 3.12, we obtain arbitrarily high energy initial data for which the solution of (1.1) blows up in finite time. 
Theorem 3.13. Assume that $\omega=0, \mu \geqslant 0$ and that (1.4) holds. Then, for every $m>0$, there exist initial data

$$
\left(u_{0}^{m}, u_{1}^{m}\right) \in \mathscr{N}_{-} \times L^{2}(\Omega)
$$

such that $\mathscr{E}\left(u_{0}^{m}, u_{1}^{m}\right) \geqslant m$ and $T_{\max }<\infty$ for the corresponding solution of (1.1).

Theorems 3.12 and 3.13 also hold for the undamped wave equation, where $\omega=\mu=0$. They are new also in this context.

Some open problems. We collect here a few questions and open problems connected with the statements of our results:

- Do Theorems 3.12 and 3.13 extend to the strongly damped case $\omega>0$ ? Also, do these results extend to the case of nonlinear (weak) damping such as $\mu\left|u_{t}\right|^{m-2} u_{t}$ with $m>2$ in place of $\mu u_{t}$ ?

- Many authors have obtained both global existence and blow up results for equations which present nonlinear damping terms such as $\left|u_{t}\right|^{m-2} u_{t}$ with $m>2$ (often enlightening the interaction that pops up with the corresponding power source $|u|^{p-2} u$ ). We refer the reader to $[6,9,13,25,26,29]$ and to the references therein. In analogy with these extensions, one could wonder whether it is possible to obtain some results for a nonlinear strong damping such as $-\Delta_{m} u_{t}$ (the $m$-Laplacian operator). We stress that our blow up Theorems 3.11, 3.12 and 3.13, being based on a kind of concavity argument (for which the linearity of the dissipation terms is particularly helpful in performing the reduction to an ordinary differential inequality in time, see e.g. (6.5)) would become too involved. Moreover, testing the equation with $u$ generates hard to manage terms which may also lack summability if $m>2$.

\section{Proof of Theorem 3.1}

We restrict ourselves to the case $\omega>0, \mu \neq 0$ and $n \geqslant 3$, the other cases being similar (and simpler), see [3]; for the case $\omega=0$ and $\mu>0$, we also refer the reader to [11].

For a given $T>0$, consider the space $\mathscr{H}=C\left([0, T], H_{0}^{1}(\Omega)\right) \cap C^{1}\left([0, T], L^{2}(\Omega)\right)$ endowed with the norm

$$
\|u\|_{\mathscr{H}}^{2}=\max _{t \in[0, T]}\left(\|\nabla u(t)\|_{2}^{2}+\left\|u_{t}(t)\right\|_{2}^{2}\right)
$$

We first prove the following

Lemma 4.1. For every $T>0$, every $u \in \mathscr{H}$ and every initial data $\left(u_{0}, u_{1}\right)$ satisfying (1.2) there exists a unique

$$
v \in \mathscr{H} \cap C^{2}\left([0, T] ; H^{-1}(\Omega)\right) \quad \text { such that } v_{t} \in L^{2}\left([0, T], H_{0}^{1}(\Omega)\right)
$$

which solves the linear problem

$$
\begin{cases}v_{t t}-\Delta v-\omega \Delta v_{t}+\mu v_{t}=|u|^{p-2} u & \text { in }[0, T] \times \Omega, \\ v(0, x)=u_{0}(x) & \text { in } \Omega, \\ v_{t}(0, x)=u_{1}(x) & \text { in } \Omega, \\ v(t, x)=0 & \text { on }[0, T] \times \partial \Omega .\end{cases}
$$

Proof. The assertion follows from an application of the Galerkin method. For every $h \geqslant 1$ let $W_{h}=\operatorname{Span}\left\{w_{1}, \ldots\right.$, $\left.w_{h}\right\}$, where $\left\{w_{j}\right\}$ is the orthogonal complete system of eigenfunctions of $-\Delta$ in $H_{0}^{1}(\Omega)$ such that $\left\|w_{j}\right\|_{2}=1$ for 
all $j$. Then, $\left\{w_{j}\right\}$ is orthogonal and complete in $L^{2}(\Omega)$ and in $H_{0}^{1}(\Omega)$; denote by $\left\{\lambda_{j}\right\}$ the related eigenvalues repeated according to their multiplicity. Let

$$
u_{0}^{h}=\sum_{j=1}^{h}\left(\int_{\Omega} \nabla u_{0} \cdot \nabla w_{j}\right) w_{j} \quad \text { and } \quad u_{1}^{h}=\sum_{j=1}^{h}\left(\int_{\Omega} u_{1} w_{j}\right) w_{j}
$$

so that $u_{0}^{h} \in W_{h}, u_{1}^{h} \in W_{h}, u_{0}^{h} \rightarrow u_{0}$ in $H_{0}^{1}(\Omega)$ and $u_{1}^{h} \rightarrow u_{1}$ in $L^{2}(\Omega)$ as $h \rightarrow \infty$. For all $h \geqslant 1$ we seek $h$ functions $\gamma_{1}^{h}, \ldots, \gamma_{h}^{h} \in C^{2}[0, T]$ such that

$$
v_{h}(t)=\sum_{j=1}^{h} \gamma_{j}^{h}(t) w_{j}
$$

solves the problem

$$
\left\{\begin{array}{l}
\int_{\Omega}\left[\ddot{v}_{h}(t)-\Delta v_{h}(t)-\omega \Delta \dot{v}_{h}(t)+\mu \dot{v}_{h}(t)-|u(t)|^{p-2} u(t)\right] \eta=0 \\
v_{h}(0)=u_{0}^{h}, \quad \dot{v}_{h}(0)=u_{1}^{h}
\end{array}\right.
$$

for every $\eta \in W_{h}$ and $t \geqslant 0$. For $j=1, \ldots, h$, taking $\eta=w_{j}$ in (4.4) yields the following Cauchy problem for a linear ordinary differential equation with unknown $\gamma_{j}^{h}$ :

$$
\left\{\begin{array}{l}
\ddot{\gamma}_{j}^{h}(t)+\left(\omega \lambda_{j}+\mu\right) \dot{\gamma}_{j}^{h}(t)+\lambda_{j} \gamma_{j}^{h}(t)=\psi_{j}(t), \\
\gamma_{j}^{h}(0)=\int_{\Omega} u_{0} w_{j}, \quad \dot{\gamma}_{j}^{h}(0)=\int_{\Omega} u_{1} w_{j}
\end{array}\right.
$$

where $\psi_{j}(t)=\int_{\Omega}|u(t)|^{p-2} u(t) w_{j} \in C[0, T]$. For all $j$, the above Cauchy problem yields a unique global solution $\gamma_{j}^{h} \in C^{2}[0, T]$. In turn, this gives a unique $v_{h}$ defined by (4.3) and satisfying (4.4). In particular, (4.3) implies that $\dot{v}_{h}(t) \in H_{0}^{1}(\Omega)$ for every $t \in[0, T]$ so that Sobolev inequality entails

$$
\left\|\dot{v}_{h}(t)\right\|_{2^{*}} \leqslant c\left\|\nabla \dot{v}_{h}(t)\right\|_{2} \quad \text { for every } t \in[0, T] .
$$

Here and in the sequel we denote by $c>0$ a generic constant that may vary even from line to line within the same formula. Taking $\eta=\dot{v}_{h}(t)$ into (4.4), and integrating over $[0, t] \subset[0, T]$, we obtain

$$
\left\|\nabla v_{h}(t)\right\|_{2}^{2}+\left\|\dot{v}_{h}(t)\right\|_{2}^{2}+2 \int_{0}^{t}\left\|\dot{v}_{h}(\tau)\right\|_{*}^{2} \mathrm{~d} \tau=\left\|\nabla u_{0}^{h}\right\|_{2}^{2}+\left\|u_{1}^{h}\right\|_{2}^{2}+2 \int_{0}^{t} \int_{\Omega}|u(\tau)|^{p-2} u(\tau) \dot{v}_{h}(\tau) \mathrm{d} \tau,
$$

for every $h \geqslant 1$. We estimate the last term in the right-hand side thanks to Hölder, Sobolev and Young inequalities (recall $p \leqslant 2^{*},(4.5)$ and $u \in C\left([0, T], H_{0}^{1}(\Omega)\right)$ ):

$$
2 \int_{0}^{t} \int_{\Omega}|u(\tau)|^{p-2} u(\tau) \dot{v}_{h}(\tau) \mathrm{d} \tau \leqslant c T+\int_{0}^{t}\left\|\dot{v}_{h}(\tau)\right\|_{*}^{2} \mathrm{~d} \tau .
$$

Recalling that $u_{0}^{h}$ and $u_{1}^{h}$ converge, from (4.6) and (4.7) we obtain

$$
\left\|v_{h}\right\|_{\mathscr{H}}^{2}+\int_{0}^{T}\left\|\dot{v}_{h}(\tau)\right\|_{*}^{2} \mathrm{~d} \tau \leqslant C_{T}
$$

for every $h \geqslant 1$, where $C_{T}>0$ is independent of $h$. By this uniform estimate and using (4.4), we have: 
$\left\{v_{h}\right\}$ is bounded in $L^{\infty}\left([0, T], H_{0}^{1}(\Omega)\right)$,

$\left\{\dot{v}_{h}\right\}$ is bounded in $L^{\infty}\left([0, T], L^{2}(\Omega)\right) \cap L^{2}\left([0, T], H_{0}^{1}(\Omega)\right)$,

$\left\{\ddot{v}_{h}\right\}$ is bounded in $L^{2}\left([0, T], H^{-1}(\Omega)\right)$;

note that $\left\{\dot{v}_{h}\right\}$ is bounded in $L^{2}\left([0, T], H_{0}^{1}(\Omega)\right)$ because we assumed $\omega>0$.

Therefore, up to a subsequence, we may pass to the limit in (4.4) and obtain a weak solution $v$ of (4.2) with the above regularity. Since $v \in H^{1}\left([0, T], H_{0}^{1}(\Omega)\right)$, we get $v \in C\left([0, T], H_{0}^{1}(\Omega)\right)$. Moreover, since $\dot{v} \in$ $L^{\infty}\left([0, T], L^{2}(\Omega)\right) \cap L^{2}\left([0, T], H_{0}^{1}(\Omega)\right)$ and $\ddot{v} \in L^{2}\left([0, T], H^{-1}(\Omega)\right)$, we have $\dot{v} \in C\left([0, T], L^{2}(\Omega)\right)$. Finally, from Eq. (4.2) we get $\ddot{v} \in C^{0}\left([0, T], H^{-1}(\Omega)\right)$. The existence of $v$ solving (4.2) and satisfying (4.1) is so proved.

Uniqueness follows arguing for contradiction: if $v$ and $w$ were two solutions of (4.2) which share the same initial data, by subtracting the equations and testing with $v_{t}-w_{t}$, instead of (4.6) we would get

$$
\|\nabla v(t)-\nabla w(t)\|_{2}^{2}+\left\|v_{t}(t)-w_{t}(t)\right\|_{2}^{2}+2 \int_{0}^{t}\left\|v_{t}(\tau)-w_{t}(\tau)\right\|_{*}^{2} \mathrm{~d} \tau=0,
$$

which immediately yields $w \equiv v$. The proof of the lemma is now complete.

Take $\left(u_{0}, u_{1}\right)$ satisfying (1.2), let $R^{2}=2\left(\left\|\nabla u_{0}\right\|_{2}^{2}+\left\|u_{1}\right\|_{2}^{2}\right)$ and for any $T>0$ consider

$$
\mathscr{M}_{T}=\left\{u \in \mathscr{H}: u(0)=u_{0}, u_{t}(0)=u_{1} \text { and }\|u\|_{\mathscr{H}} \leqslant R\right\} .
$$

By Lemma 4.1, for any $u \in \mathscr{M}_{T}$ we may define $v=\Phi(u)$, being $v$ the unique solution to problem (4.2). We claim that, for a suitable $T>0, \Phi$ is a contractive map satisfying $\Phi\left(\mathscr{M}_{T}\right) \subseteq \mathscr{M}_{T}$. Given $u \in \mathscr{M}_{T}$, the corresponding solution $v=\Phi(u)$ satisfies for all $t \in(0, T]$ the energy identity (see (4.6)):

$$
\|\nabla v(t)\|_{2}^{2}+\left\|v_{t}(t)\right\|_{2}^{2}+2 \int_{0}^{t}\left\|v_{t}(\tau)\right\|_{*}^{2} \mathrm{~d} \tau=\left\|\nabla u_{0}\right\|_{2}^{2}+\left\|u_{1}\right\|_{2}^{2}+2 \int_{0}^{t} \int_{\Omega}|u(\tau)|^{p-2} u(\tau) v_{t}(\tau) \mathrm{d} \tau .
$$

For the last term, we argue in the same spirit (although slightly differently) as for (4.7) and we get (recall $\omega>0$ )

$$
\begin{aligned}
2 \int_{0}^{t} \int_{\Omega}|u(\tau)|^{p-2} u(\tau) v_{t}(\tau) \mathrm{d} \tau & \leqslant c \int_{0}^{T}\|u(\tau)\|_{2^{*}}^{p-1}\left\|v_{t}(\tau)\right\|_{2^{*}} \mathrm{~d} \tau \leqslant c \int_{0}^{T}\|u(\tau)\|_{*}^{p-1}\left\|v_{t}(\tau)\right\|_{*} \mathrm{~d} \tau \\
& \leqslant c T R^{2(p-1)}+2 \int_{0}^{T}\left\|v_{t}(\tau)\right\|_{*}^{2} \mathrm{~d} \tau
\end{aligned}
$$

for all $t \in(0, T]$. Combining (4.8) with (4.9) and taking the maximum over $[0, T]$ gives

$$
\|v\|_{\mathscr{H}}^{2} \leqslant \frac{1}{2} R^{2}+c T R^{2(p-1)} .
$$

Choosing $T$ sufficiently small, we get $\|v\|_{\mathscr{H}} \leqslant R$, which shows that $\Phi\left(\mathscr{M}_{T}\right) \subseteq \mathscr{M}_{T}$. Now, take $w_{1}$ and $w_{2}$ in $\mathscr{M}_{T}$; subtracting the two equations (4.2) for $v_{1}=\Phi\left(w_{1}\right)$ and $v_{2}=\Phi\left(w_{2}\right)$, and setting $v=v_{1}-v_{2}$ we obtain for all $\eta \in H_{0}^{1}(\Omega)$ and a.e. $t \in[0, T]$

$$
\begin{aligned}
\left\langle v_{t t}(t), \eta\right\rangle+\int_{\Omega} \nabla v(t) \cdot \nabla \eta+\omega \int_{\Omega} \nabla v_{t}(t) \cdot \nabla \eta+\mu \int_{\Omega} v_{t}(t) \eta & =\int_{\Omega}\left(\left|w_{1}(t)\right|^{p-2} w_{1}(t)-\left|w_{2}(t)\right|^{p-2} w_{2}(t)\right) \eta \\
& =\int_{\Omega} \xi(t)\left(w_{1}(t)-w_{2}(t)\right) \eta
\end{aligned}
$$


where $\xi=\xi(x, t) \geqslant 0$ is given by Lagrange Theorem so that $\xi(t) \leqslant(p-1)\left(\left|w_{1}(t)\right|+\left|w_{2}(t)\right|\right)^{p-2}$. Therefore, by taking $\eta=v_{t}$ in (4.10) and arguing as above, we obtain

$$
\left\|\Phi\left(w_{1}\right)-\Phi\left(w_{2}\right)\right\|_{\mathscr{H}}^{2}=\|v\|_{\mathscr{H}}^{2} \leqslant c R^{2 p-4} T\left\|w_{1}-w_{2}\right\|_{\mathscr{H}}^{2} \leqslant \delta\left\|w_{1}-w_{2}\right\|_{\mathscr{H}}^{2}
$$

for some $\delta<1$ provided $T$ is sufficiently small. This proves the claim. By the Contraction Mapping Principle, there exists a unique (weak) solution to (1.1) defined on $[0, T]$. The main statement of Theorem 3.1 is so proved.

Concerning the last assertion we observe that, by the construction above, once $\omega>0$ is fixed, the local existence time of $u$ merely depends (through $R$ ) on the norms of the initial data. Therefore, as long as $\|u(t)\|_{\mathscr{H}}$ remains bounded, the solution may be continued, see also [22, p. 158] for a similar argument. Hence, if $T_{\max }<\infty$, we have

$$
\lim _{t \rightarrow T_{\max }}\|\nabla u(t)\|_{2}^{2}+\left\|u_{t}(t)\right\|_{2}^{2}=\lim _{t \rightarrow T_{\max }}\|u(t)\|_{\mathscr{H}}^{2}=\infty .
$$

Consider the energy function

$$
E(t)=\frac{1}{2}\|\nabla u(t)\|_{2}^{2}+\frac{1}{2}\left\|u_{t}(t)\right\|_{2}^{2}-\frac{1}{p}\|u(t)\|_{p}^{p}, \quad t \in\left[0, T_{\max }\right),
$$

which satisfies

$$
E(t)+\int_{s}^{t}\left\|u_{t}(\tau)\right\|_{*}^{2} \mathrm{~d} \tau=E(s) \quad \text { for every } 0 \leqslant s \leqslant t<T_{\max } .
$$

Since $\omega>0$, this energy identity follows by testing (1.1) with $u_{t}$ and integrating with respect to $t$. Note that (4.13) also holds in the case $\omega=0$, see [9, Proposition 2.1]. In both the cases, by (4.13), the map $\{t \mapsto E(t)\}$ is nonincreasing. As a consequence,

$$
\frac{1}{2}\|\nabla u(t)\|_{2}^{2}+\frac{1}{2}\left\|u_{t}(t)\right\|_{2}^{2} \leqslant \frac{1}{p}\|u(t)\|_{p}^{p}+E(0) \quad \text { for all } t \in\left[0, T_{\max }\right)
$$

which, together with (4.11), implies

$$
\lim _{t \rightarrow T_{\max }}\|u(t)\|_{p}=\infty
$$

This proves at once the very last statement of Theorem 3.1 when $p=2^{*}=q=\frac{n(p-2)}{2}$. For the remaining cases, notice first that (4.15) implies

$$
\lim _{t \rightarrow T_{\max }}\|\nabla u(t)\|_{2}=\infty .
$$

Moreover, by (4.14) we obtain

$$
\|\nabla u(t)\|_{2}^{2} \leqslant 2 E(0)+\frac{2}{p}\|u(t)\|_{p}^{p}, \quad t \in\left[0, T_{\max }\right)
$$

which, combined with the Gagliardo-Nirenberg inequality, yields:

$$
c\|\nabla u(t)\|_{2}^{2}-c \leqslant\|u(t)\|_{p}^{p} \leqslant c\|u(t)\|_{q}^{p(1-\sigma)}\|\nabla u(t)\|_{2}^{p \sigma} \quad \text { for } \sigma=\frac{2 n(p-q)}{p(2 n+2 q-n q)} .
$$

Since $n(p-2) / 2<q<p$ implies $\sigma \in(0,1)$ and $p \sigma<2$, the above inequality combined with (4.16) immediately yields (3.1). This completes the proof of Theorem 3.1. 


\section{Proof of Theorem 3.8}

Throughout the proof we denote by $c>0$ a generic constant, independent of $\omega$, possibly dependent on $\mu$ and on the initial data $\left(u_{0}, u_{1}\right)$, that may vary even from line to line within the same formula.

Consider the case $\omega>0$ and $\mu>-\lambda_{1} \omega$. Without loss of generality, we may assume that $\bar{t}=0$. By (4.13) we know that the energy map $E$ is decreasing. Then, if condition (3.8) holds true, we have

$$
u(t) \in \mathscr{W} \quad \text { and } \quad E(t)<d \quad \text { for every } t \in\left(0, T_{\max }\right) .
$$

Indeed, if it was not the case, there would exist $t_{*}>0$ such that $u\left(t_{*}\right) \in \mathscr{N}$. By the variational characterization (2.3) of $d$,

$$
d \leqslant J\left(u\left(t_{*}\right)\right) \leqslant E\left(t_{*}\right)<d,
$$

a contradiction to (5.1). As a further consequence of (5.1), a simple computation entails

$$
J(u(t)) \geqslant \frac{p-2}{2 p}\|\nabla u(t)\|_{2}^{2} \quad \text { for every } t \in\left[0, T_{\max }\right) .
$$

For all $t \in\left[0, T_{\max }\right)$, by (4.13) we obtain

$$
\frac{1}{2}\left\|u_{t}(t)\right\|_{2}^{2}+J(u(t))+\int_{0}^{t}\left\|u_{t}(\tau)\right\|_{*}^{2} \mathrm{~d} \tau=E(0) \leqslant d .
$$

Therefore, by virtue of (5.2) the Continuation Principle yields $T_{\max }=\infty$ and

$$
\begin{aligned}
& \|\nabla u(t)\|_{2}^{2}+\left\|u_{t}(t)\right\|_{2}^{2} \leqslant c \quad \text { for every } t \in[0, \infty), \\
& \int_{0}^{t}\left\|\nabla u_{t}(\tau)\right\|_{2}^{2} \mathrm{~d} \tau \leqslant \frac{c}{\omega} \quad \text { for every } t \in[0, \infty) .
\end{aligned}
$$

Hence, by Poincaré inequality, we get

$$
\int_{0}^{t}\left\|u_{t}(\tau)\right\|_{2}^{2} \mathrm{~d} \tau \leqslant \frac{c}{\omega} \quad \text { for every } t \in[0, \infty)
$$

Now, as in [13], we integrate over $[0, t]$ the trivial inequality

$$
\frac{\mathrm{d}}{\mathrm{d} t}((1+t) E(t)) \leqslant E(t)
$$

and recalling that by [14, Lemma 5.2] there holds

$$
J(u(t)) \leqslant c I(u(t)) \text { for every } t \in[0, \infty),
$$

we reach the inequality

$$
(1+t) E(t) \leqslant d+\frac{1}{2} \int_{0}^{t}\left\|u_{t}(\tau)\right\|_{2}^{2} \mathrm{~d} \tau+c \int_{0}^{t} I(u(\tau)) \mathrm{d} \tau
$$

for every $t \in[0, \infty)$. Observe also that, by direct computation, there holds

$$
\left\langle u_{t t}(t), u(t)\right\rangle=\frac{\mathrm{d}}{\mathrm{d} t} \int_{\Omega} u_{t}(t) u(t)-\left\|u_{t}(t)\right\|_{2}^{2} \text { for a.e. } t \in[0, \infty) .
$$


Moreover, by testing the equation with $u$, we obtain

$$
\left\langle u_{t t}(t), u(t)\right\rangle+\|\nabla u(t)\|_{2}^{2}+\left(u(t), u_{t}(t)\right)_{*}=\|u(t)\|_{p}^{p} \quad \text { for a.e. } t \in[0, \infty) .
$$

Using (5.6), this yields

$$
\frac{\mathrm{d}}{\mathrm{d} t}\left(\int_{\Omega} u u_{t}+\frac{1}{2}\|u\|_{*}^{2}\right)=\left\|u_{t}\right\|_{2}^{2}-I(u)
$$

By integrating (5.7) on $[0, t]$ and by (5.3) and (5.4), we have

$$
\begin{aligned}
\int_{0}^{t} I(u(\tau)) \mathrm{d} \tau & \leqslant \int_{0}^{t}\left\|u_{t}(\tau)\right\|_{2}^{2} \mathrm{~d} \tau+\left\|u_{1}\right\|_{2}\left\|u_{0}\right\|_{2}+\left\|u_{t}(t)\right\|_{2}\|u(t)\|_{2}+\frac{1}{2}\left(\left\|u_{0}\right\|_{*}^{2}-\|u(t)\|_{*}^{2}\right) \\
& \leqslant c+\frac{c}{\omega}+c \omega
\end{aligned}
$$

for every $t \in[0, \infty)$. Then, by combining the above inequalities, from (5.5) we get

$$
E(t) \leqslant c\left(1+\frac{1}{\omega}+\omega\right) \frac{1}{t}
$$

for every $t \in(0, \infty)$. Consequently, by (5.2) we immediately obtain

$$
\|\nabla u(t)\|_{2}^{2}+\left\|u_{t}(t)\right\|_{2}^{2} \leqslant \frac{\Theta(\omega, \mu)}{t},
$$

for every $t \in(0, \infty)$, where $\Theta$ is the map defined in (3.10). The proof in the case $\omega=0$ (and $\mu>0$ ) is similar and follows by obvious modifications of inequalities (5.4) and (5.8).

\section{Proof of Theorem 3.11}

Assume first that there exists $\bar{t} \geqslant 0$ such that $u(\bar{t}) \in \mathscr{U}$ and $\mathscr{E}\left(u(\bar{t}), u_{t}(\bar{t})\right) \leqslant d$. Without loss of generality, we may assume that $\bar{t}=0$ so that $\left(u(\bar{t}), u_{t}(\bar{t})\right)=\left(u_{0}, u_{1}\right)$. By (4.13) we know that $E(t)<d$ for all $t>0$ and therefore $u(t) \notin \mathscr{N}$. This shows that $u(t) \in \mathscr{U}$ for all $t \in\left[0, T_{\max }\right)$. Hence, by (2.4) we obtain

$$
\|\nabla u(t)\|_{2}^{2}>\frac{2 d p}{p-2} \quad \text { for every } t \in\left[0, T_{\max }\right) .
$$

Assume by contradiction that the solution $u$ is global. Then, for any $T>0$ we may consider $\theta:[0, T] \rightarrow \mathbb{R}_{+}$ defined by

$$
\theta(t)=\|u(t)\|_{2}^{2}+\int_{0}^{t}\|u(\tau)\|_{*}^{2} \mathrm{~d} \tau+(T-t)\left\|u_{0}\right\|_{*}^{2} .
$$

Notice $\theta(t)>0$ for all $t \in[0, T]$; hence, since $\theta$ is continuous, there exists $\rho>0$ (independent of the choice of $T$ ) such that

$$
\theta(t) \geqslant \rho \quad \text { for all } t \in[0, T]
$$

furthermore,

$$
\theta^{\prime}(t)=2 \int_{\Omega} u(t) u_{t}(t)+\|u(t)\|_{*}^{2}-\left\|u_{0}\right\|_{*}^{2}=2 \int_{\Omega} u(t) u_{t}(t)+2 \int_{0}^{t}\left(u(\tau), u_{t}(\tau)\right)_{*} \mathrm{~d} \tau
$$


and, consequently, using (5.6)

$$
\theta^{\prime \prime}(t)=2\left\langle u_{t t}(t), u(t)\right\rangle+2\left\|u_{t}(t)\right\|_{2}^{2}+2\left(u(t), u_{t}(t)\right)_{*} \quad \text { for a.e. } t \in[0, T] .
$$

Testing the equation in (1.1) with $u$ and plugging the result into the expression of $\theta^{\prime \prime}$ we obtain

$$
\theta^{\prime \prime}(t)=2\left(\left\|u_{t}(t)\right\|_{2}^{2}-\|\nabla u(t)\|_{2}^{2}+\|u(t)\|_{p}^{p}\right) \quad \text { for a.e. } t \in[0, T] .
$$

Therefore, we get

$$
\begin{aligned}
\theta(t) \theta^{\prime \prime}(t)-\frac{p+2}{4} \theta^{\prime}(t)^{2}= & 2 \theta(t)\left\{\left\|u_{t}(t)\right\|_{2}^{2}-\|\nabla u(t)\|_{2}^{2}+\|u(t)\|_{p}^{p}\right\} \\
& +(p+2)\left\{\eta(t)-\left\{\theta(t)-(T-t)\left\|u_{0}\right\|_{*}^{2}\right\}\left(\left\|u_{t}(t)\right\|_{2}^{2}+\int_{0}^{t}\left\|u_{t}(\tau)\right\|_{*}^{2} \mathrm{~d} \tau\right)\right\},
\end{aligned}
$$

where $\eta:[0, T] \rightarrow \mathbb{R}_{+}$is the function defined by

$$
\eta(t)=\left(\|u(t)\|_{2}^{2}+\int_{0}^{t}\|u(\tau)\|_{*}^{2} \mathrm{~d} \tau\right)\left(\left\|u_{t}(t)\right\|_{2}^{2}+\int_{0}^{t}\left\|u_{t}(\tau)\right\|_{*}^{2} \mathrm{~d} \tau\right)-\left(\int_{\Omega} u(t) u_{t}(t)+\int_{0}^{t}\left(u(\tau), u_{t}(\tau)\right)_{*} \mathrm{~d} \tau\right)^{2} .
$$

Notice that, using Schwarz inequality, we obtain

$$
\begin{aligned}
& \|u(t)\|_{2}^{2}\left\|u_{t}(t)\right\|_{2}^{2} \geqslant\left(\int_{\Omega} u(t) u_{t}(t)\right)^{2}, \\
& \int_{0}^{t}\|u(\tau)\|_{*}^{2} \mathrm{~d} \tau \int_{0}^{t}\left\|u_{t}(\tau)\right\|_{*}^{2} \mathrm{~d} \tau \geqslant\left(\int_{0}^{t}\left(u(\tau), u_{t}(\tau)\right)_{*} \mathrm{~d} \tau\right)^{2},
\end{aligned}
$$

and

$$
\int_{\Omega} u(t) u_{t}(t) \int_{0}^{t}\left(u(\tau), u_{t}(\tau)\right)_{*} \mathrm{~d} \tau \leqslant\|u(t)\|_{2}\left(\int_{0}^{t}\left\|u_{t}(\tau)\right\|_{*}^{2} \mathrm{~d} \tau\right)^{1 / 2}\left\|u_{t}(t)\right\|_{2}\left(\int_{0}^{t}\|u(\tau)\|_{*}^{2} \mathrm{~d} \tau\right)^{1 / 2} .
$$

These three inequalities entail $\eta(t) \geqslant 0$ for every $t \in[0, T]$. As a consequence, we reach the following differential inequality

$$
\theta(t) \theta^{\prime \prime}(t)-\frac{p+2}{4} \theta^{\prime}(t)^{2} \geqslant \theta(t) \xi(t) \quad \text { for a.e. } t \in[0, T],
$$

where $\xi:[0, T] \rightarrow \mathbb{R}_{+}$is the map defined by

$$
\xi(t)=-2 p E(t)+(p-2)\|\nabla u(t)\|_{2}^{2}-(p+2) \int_{0}^{t}\left\|u_{t}(\tau)\right\|_{*}^{2} \mathrm{~d} \tau
$$

By (4.13), for all $t \in[0, T]$ we may also write

$$
\xi(t)=-2 p E(0)+(p-2)\|\nabla u(t)\|_{2}^{2}+(p-2) \int_{0}^{t}\left\|u_{t}(\tau)\right\|_{*}^{2} \mathrm{~d} \tau
$$

and therefore, by (6.1), we obtain 


$$
\begin{aligned}
\xi(t) & =\xi(0)+(p-2)\|\nabla u(t)\|_{2}^{2}-(p-2)\left\|\nabla u_{0}\right\|_{2}^{2}+(p-2) \int_{0}^{t}\left\|u_{t}(\tau)\right\|_{*}^{2} \mathrm{~d} \tau \\
& >2 p(d-E(0))+(p-2) \int_{0}^{t}\left\|u_{t}(\tau)\right\|_{*}^{2} \mathrm{~d} \tau \geqslant(p-2) \int_{0}^{t}\left\|u_{t}(\tau)\right\|_{*}^{2} \mathrm{~d} \tau \geqslant 0
\end{aligned}
$$

since $E(0) \leqslant d$. Hence, there exists $\delta>0$ (independent of $T$ ) such that

$$
\xi(t) \geqslant \delta \quad \text { for all } t \geqslant 0 .
$$

By (6.2), (6.3) and (6.4) it follows that

$$
\theta(t) \theta^{\prime \prime}(t)-\frac{p+2}{4} \theta^{\prime}(t)^{2} \geqslant \rho \delta \quad \text { for a.e. } t \in[0, T] .
$$

Setting $y(t)=\theta(t)^{-(p-2) / 4}$, this inequality becomes

$$
y^{\prime \prime}(t) \leqslant-\frac{p-2}{4} \rho \delta y(t) \quad \text { for a.e. } t \in[0, T] .
$$

This proves that $y(t)$ reaches 0 in finite time, say as $t \rightarrow T^{*}$. Since $T^{*}$ is independent of the initial choice of $T$, we may assume that $T^{*}<T$. This tells us that

$$
\lim _{t \rightarrow T^{*}} \theta(t)=\infty
$$

In turn, this implies that

$$
\lim _{t \rightarrow T^{*}}\|\nabla u(t)\|_{2}^{2}=\infty .
$$

Indeed, if $\|u(t)\|_{2} \rightarrow \infty$ as $t \rightarrow T^{*}$, then (6.6) immediately follows. On the contrary, if $\|u(t)\|_{2}$ remains bounded on $\left[0, T^{*}\right)$, then

$$
\lim _{t \rightarrow T^{*}} \int_{0}^{t}\|u(\tau)\|_{*}^{2} \mathrm{~d} \tau=\infty
$$

so that again (6.6) is satisfied.

Conversely, assume now that $T_{\max }<\infty$. Notice first that, for every $t>0$, there holds

$$
\int_{0}^{t}\left\|u_{t}(\tau)\right\|_{*}^{2} \mathrm{~d} \tau \geqslant \frac{1}{t}\left(\int_{0}^{t}\left\|u_{t}(\tau)\right\|_{*} \mathrm{~d} \tau\right)^{2} \geqslant \frac{1}{t}\left(\|u(t)\|_{*}-\left\|u_{0}\right\|_{*}\right)^{2} .
$$

Hence, by (4.13), we obtain

$$
\frac{1}{2} I(u(t)) \leqslant E(t) \leqslant E(0)-\frac{1}{t}\left(\|u(t)\|_{*}-\left\|u_{0}\right\|_{*}\right)^{2} .
$$

Since $\|u(t)\|_{*} \rightarrow \infty$ as $t \rightarrow T_{\max }$, we conclude that

$$
\lim _{t \rightarrow T_{\max }} I(u(t))=\lim _{t \rightarrow T_{\max }} E(t)=-\infty .
$$

Then, the desired assertion immediately follows. 


\section{Proof of Theorems 3.4 and 3.6}

Assume that $u=u(t)$ is a global solution to (1.1) and (1.2) and let $E: \mathbb{R}_{+} \rightarrow \mathbb{R}$ be its energy as defined in (4.12). Without loss of generality, we may assume that

$$
d \leqslant E(t) \leqslant E(0) \quad \text { for every } t \in \mathbb{R}_{+} .
$$

Indeed, the right-hand side inequality holds true since the map $\{t \mapsto E(t)\}$ is nonincreasing in view of (4.13). On the other hand, if it was $E\left(t_{0}\right)<d$ for some $t_{0}>0$, taking into account Theorem 3.8 and Theorem 3.11 (proved above!), either $\|\nabla u(t)\|_{2}^{2}+\left\|u_{t}(t)\right\|_{2}^{2} \rightarrow 0$ as $t \rightarrow \infty$ if $u\left(t_{0}\right) \in \mathscr{W}$ (in which case Theorems 3.4 and 3.6 would automatically hold true) or $u$ is not global if $u\left(t_{0}\right) \in \mathscr{U}$, against the assumption.

Before starting the proof of Theorems 3.4 and 3.6, some preliminary facts are in order. Firstly we prove a global summability property for $u_{t}$. For simplicity we assume that $\omega>0$, the case $\omega=0$ being similar. Taking into account that $u_{t}(\tau) \in H_{0}^{1}(\Omega)$ for a.e. $\tau \geqslant 0$, we combine Poincaré inequality with (4.13) and (7.1) to show that, for every $t>0$ we have

$$
\int_{0}^{t}\left\|u_{t}(\tau)\right\|_{2}^{2} \mathrm{~d} \tau \leqslant c \int_{0}^{t}\left\|u_{t}(\tau)\right\|_{*}^{2} \mathrm{~d} \tau \leqslant c(E(0)-d) .
$$

Letting $t \rightarrow \infty$, we conclude that

$$
\int_{0}^{\infty}\left\|u_{t}(\tau)\right\|_{2}^{2} \mathrm{~d} \tau<\infty \quad \text { if } \omega \geqslant 0, \quad \int_{0}^{\infty}\left\|\nabla u_{t}(\tau)\right\|_{2}^{2} \mathrm{~d} \tau<\infty \quad \text { if } \omega>0 .
$$

Furthermore, observe that by the definition of $E(t)$ and (7.1), we obtain

$$
\|u(t)\|_{p}^{p} \geqslant \frac{p}{2}\|\nabla u(t)\|_{2}^{2}-p E(0) \quad \text { for every } t \in \mathbb{R}_{+} .
$$

Then, plugging this inequality into identity (5.7) yields

$$
\frac{\mathrm{d}}{\mathrm{d} t}\left(\int_{\Omega} u(t) u_{t}(t)+\frac{1}{2}\|u(t)\|_{*}^{2}\right) \geqslant \frac{p-2}{2}\|\nabla u(t)\|_{2}^{2}-p E(0) .
$$

Inspired by [8] we now prove a crucial stability result.

Lemma 7.1. Under the assumptions of Theorems 3.4 and 3.6, for every $\kappa>0$ we have

$$
\begin{aligned}
& \lim _{t \rightarrow \infty}\|\nabla u(t)-\nabla u(t+\kappa)\|_{2}=0 \quad \text { for } \omega>0, \\
& \lim _{t \rightarrow \infty}\|u(t)-u(t+\kappa)\|_{2}=0 \quad \text { for } \omega=0 .
\end{aligned}
$$

Proof. Let $\omega>0$. Fixed $\kappa>0$, by (4.13), for every $t>0$ we have

$$
\begin{aligned}
\int_{\Omega}|\nabla u(t)-\nabla u(t+\kappa)|^{2} & =\int_{\Omega}\left|\int_{t}^{t+\kappa} \nabla u_{t}(\tau) \mathrm{d} \tau\right|^{2} \leqslant \kappa \int_{\Omega} \int_{t}^{t+\kappa}\left|\nabla u_{t}(\tau)\right|^{2} \mathrm{~d} \tau \\
& \leqslant \kappa c \int_{t}^{t+\kappa}\left\|u_{t}(\tau)\right\|_{*}^{2} \mathrm{~d} \tau=\kappa c(E(t)-E(t+\kappa)) .
\end{aligned}
$$

Since $E(t)$ is nonincreasing and lower bounded by (7.1), $E(t)$ admits finite limit as $t \rightarrow \infty$. This immediately yields the assertion by letting $t \rightarrow \infty$ in the previous inequality. The proof in the case $\omega=0$ is similar. 


\subsection{Proof of Theorem 3.4}

Assume by contradiction that (3.2) fails, namely that there exists a diverging sequence $\left\{t_{j}\right\} \subset \mathbb{R}_{+}$such that

$$
\left\|u_{t}\left(t_{j}\right)\right\|_{2}^{2}+\left\|\nabla u\left(t_{j}\right)\right\|_{2}^{2} \rightarrow \infty \quad \text { as } j \rightarrow \infty .
$$

Then, by (7.1) we have $\left\|u\left(t_{j}\right)\right\|_{p} \rightarrow \infty$ so that by Sobolev inequality

$$
\left\|\nabla u\left(t_{j}\right)\right\|_{2} \rightarrow \infty \quad \text { as } j \rightarrow \infty \text {. }
$$

By (7.5) and continuity, we can select a diverging sequence $\left\{\bar{t}_{m}\right\} \subset \mathbb{R}_{+}$such that $\left\|u\left(\bar{t}_{m}\right)\right\|_{*}^{2}=m^{2}+1$. Moreover, since Lemma 7.1 with $\omega>0$ rephrases as

$$
\lim _{\tau \rightarrow \infty} \sup \left\{\kappa>0:\|u(t)-u(t+\kappa)\|_{*}<1, \forall t \geqslant \tau\right\}=\infty
$$

we find a second diverging sequence $\left\{\tau_{m}\right\} \subset \mathbb{R}_{+}$such that

$$
m^{2} \leqslant\|u(t)\|_{*}^{2} \leqslant m^{2}+2 \text { for every } t \in\left[\bar{t}_{m}, \bar{t}_{m}+\tau_{m}\right] .
$$

In view of (7.2), for all $m$ sufficiently large,

$$
\text { there exists } t_{m} \in\left[\bar{t}_{m}, \bar{t}_{m}+1\right] \text { such that }\left\|u_{t}\left(t_{m}\right)\right\|_{2}^{2}<2 d \text {. }
$$

Clearly, up to renaming $\tau_{m}$ into $\left(\tau_{m}-1\right)$ we now have

$$
m^{2} \leqslant\|u(t)\|_{*}^{2} \leqslant m^{2}+2 \text { for every } t \in\left[t_{m}, t_{m}+\tau_{m}\right] .
$$

Also, for $m$ large enough, there holds

$$
\int_{\Omega} u\left(t_{m}\right) u_{t}\left(t_{m}\right)+\frac{1}{2}\left\|u\left(t_{m}\right)\right\|_{*}^{2} \geqslant 0 .
$$

Indeed, by (7.6), (7.7), Young, Hölder, and Poincaré inequalities,

$$
\begin{aligned}
\int_{\Omega} u\left(t_{m}\right) u_{t}\left(t_{m}\right)+\frac{1}{2}\left\|u\left(t_{m}\right)\right\|_{*}^{2} & \geqslant \frac{1}{2}\left\|u\left(t_{m}\right)\right\|_{*}^{2}-\left\|u\left(t_{m}\right)\right\|_{2}\left\|u_{t}\left(t_{m}\right)\right\|_{2} \\
& \geqslant \frac{1}{4}\left\|u\left(t_{m}\right)\right\|_{*}^{2}-c\left\|u_{t}\left(t_{m}\right)\right\|_{2}^{2} \geqslant \frac{m^{2}}{4}-2 c d \geqslant 0
\end{aligned}
$$

for every $m$ large enough.

By (7.9) and integrating (7.3) on the time interval $\left[t_{m}, t\right]$ for $t \in\left(t_{m}, t_{m}+\tau_{m}\right]$ entails

$$
\int_{\Omega} u(t) u_{t}(t)+\frac{1}{2}\|u(t)\|_{*}^{2} \geqslant \int_{t_{m}}^{t}\left(\frac{p-2}{2}\|\nabla u(\tau)\|_{2}^{2}-p E(0)\right) \mathrm{d} \tau \geqslant c \int_{t_{m}}^{t}\|u(\tau)\|_{*}^{2} \mathrm{~d} \tau
$$

provided $m$ is sufficiently large. On the other hand, by Hölder, Young and Poincaré inequalities,

$$
\int_{\Omega} u(t) u_{t}(t)+\frac{1}{2}\|u(t)\|_{*}^{2} \leqslant\|u(t)\|_{2}\left\|u_{t}(t)\right\|_{2}+\frac{1}{2}\|u(t)\|_{*}^{2} \leqslant \frac{1}{2}\left\|u_{t}(t)\right\|_{2}^{2}+c\|u(t)\|_{*}^{2} .
$$

Summarizing, if we set

$$
\Upsilon_{m}(t)=\int_{t_{m}}^{t}\|u(\tau)\|_{*}^{2} \mathrm{~d} \tau \quad \text { for every } t \in\left(t_{m}, t_{m}+\tau_{m}\right]
$$


then the following differential inequality is satisfied

$$
\Upsilon_{m}^{\prime}(t) \geqslant \gamma \Upsilon_{m}(t)-C\left\|u_{t}(t)\right\|_{2}^{2} \text { for every } t \in\left(t_{m}, t_{m}+\tau_{m}\right],
$$

for some $\gamma>0$ and $C>0$; hence,

$$
\frac{\Upsilon_{m}^{\prime}(t)}{\Upsilon_{m}(t)} \geqslant \gamma-C \frac{\left\|u_{t}(t)\right\|_{2}^{2}}{\Upsilon_{m}(t)} \quad \text { for every } t \in\left(t_{m}, t_{m}+\tau_{m}\right] .
$$

Notice that, since by (7.8) we have

$$
\Upsilon_{m}(t) \geqslant m^{2}\left(t-t_{m}\right) \geqslant \frac{m^{2} \tau_{m}}{2} \quad \text { for every } t \in\left[t_{m}+\frac{\tau_{m}}{2}, t_{m}+\tau_{m}\right],
$$

the differential inequality (7.10) yields

$$
\frac{\Upsilon_{m}^{\prime}(t)}{\Upsilon_{m}(t)} \geqslant \gamma-2 C \frac{\left\|u_{t}(t)\right\|_{2}^{2}}{m^{2} \tau_{m}} \quad \text { for every } t \in\left[t_{m}+\frac{\tau_{m}}{2}, t_{m}+\tau_{m}\right] .
$$

Integrating (7.12) over $\left[t_{m}+\frac{\tau_{m}}{2}, t_{m}+\tau_{m}\right]$ and taking into account (7.2) we find

$$
\log \Upsilon_{m}\left(t_{m}+\tau_{m}\right) \geqslant \log \Upsilon_{m}\left(t_{m}+\frac{\tau_{m}}{2}\right)+\gamma \frac{\tau_{m}}{2}-\frac{2 C \alpha}{m^{2} \tau_{m}}
$$

where we have set $\alpha=\int_{0}^{\infty}\left\|u_{t}(\tau)\right\|_{2}^{2} \mathrm{~d} \tau$. Hence, up to enlarging $m$, we may take the exponential and we finally conclude that

$$
\int_{t_{m}}^{t_{m}+\tau_{m}}\|u(\tau)\|_{*}^{2} \mathrm{~d} \tau=\Upsilon_{m}\left(t_{m}+\tau_{m}\right) \geqslant \frac{1}{2} \Upsilon_{m}\left(t_{m}+\frac{\tau_{m}}{2}\right) \mathrm{e}^{\gamma \tau_{m} / 2} \geqslant \frac{m^{2} \tau_{m}}{4} \mathrm{e}^{\gamma \tau_{m} / 2}
$$

where we also used (7.11). On the other hand, by inequality (7.8), it turns out that

$$
\int_{t_{m}}^{t_{m}+\tau_{m}}\|u(\tau)\|_{*}^{2} \mathrm{~d} \tau \leqslant\left(m^{2}+2\right) \tau_{m}
$$

which contradicts (7.13), since $\tau_{m} \rightarrow \infty$. Therefore, (7.4) is false and $\{u(t)\}$ is bounded, namely there exists $c>0$ such that

$$
\left\|u_{t}(t)\right\|_{2}^{2}+\|\nabla u(t)\|_{2}^{2} \leqslant c \quad \text { for all } t \geqslant 0 .
$$

We now turn to the proof of the second part of Theorem 3.4 for $n=1,2$ or under assumption (3.3). Since $u_{t} \in C^{0}\left(\mathbb{R}_{+}, L^{2}(\Omega)\right)$, by (7.2) there exist a diverging sequence $\left\{t_{j}\right\} \subset \mathbb{R}_{+}, \varepsilon \in(0,1)$ and a sequence $\left\{\varepsilon_{j}\right\} \subset[\varepsilon, 1]$ such that

$$
\lim _{j \rightarrow \infty} \int_{t_{j}}^{t_{j}+\varepsilon_{j}}\left\|u_{t}(\tau)\right\|_{*}^{2} \mathrm{~d} \tau=0, \quad \lim _{j \rightarrow \infty}\left\|u_{t}\left(t_{j}\right)\right\|_{2}+\left\|u_{t}\left(t_{j}+\varepsilon_{j}\right)\right\|_{2}=0 .
$$

In particular, from (7.15), for every $\eta \in H_{0}^{1}(\Omega)$ there holds

$$
\int_{t_{j}}^{t_{j}+\varepsilon_{j}}\left\langle u_{t t}(\tau), \eta\right\rangle \mathrm{d} \tau=\int_{\Omega} u_{t}\left(t_{j}+\varepsilon\right) \eta-\int_{\Omega} u_{t}\left(t_{j}\right) \eta=\mathrm{o}(1) \quad \text { as } j \rightarrow \infty .
$$

Integrating Eq. (1.1) over $\left[t_{j}, t_{j}+\varepsilon_{j}\right]$ and using again (7.15) yields 


$$
\begin{aligned}
& \int_{t_{j}}^{t_{j}+\varepsilon_{j}}\left(\int_{\Omega}\left(\nabla u(\tau) \cdot \nabla \eta-|u(\tau)|^{p-2} u(\tau) \eta\right)\right) \mathrm{d} \tau \\
& \quad=-\int_{t_{j}}^{t_{j}+\varepsilon_{j}}\left\langle u_{t t}(\tau), \eta\right\rangle \mathrm{d} \tau-\int_{t_{j}}^{t_{j}+\varepsilon_{j}}\left(u_{t}(\tau), \eta\right)_{*} \mathrm{~d} \tau=\mathrm{o}(1) \quad \text { as } j \rightarrow \infty
\end{aligned}
$$

for every $\eta \in H_{0}^{1}(\Omega)$. Consequently, recalling that $u \in C^{0}\left(\mathbb{R}_{+}, H_{0}^{1}(\Omega)\right)$, for any such $\eta$ and for any $j$ we may find $t_{j}^{\eta} \in\left[t_{j}, t_{j}+\varepsilon_{j}\right]$ such that

$$
\lim _{j \rightarrow \infty} \int_{\Omega}\left(\nabla u\left(t_{j}^{\eta}\right) \cdot \nabla \eta-\left|u\left(t_{j}^{\eta}\right)\right|^{p-2} u\left(t_{j}^{\eta}\right) \eta\right)=0 ;
$$

in fact, by (7.14) and Lemma 7.1 we may take $t_{j}^{\eta}=t_{j}$ for any $\eta$, namely

$$
\lim _{j \rightarrow \infty} \int_{\Omega}\left(\nabla u\left(t_{j}\right) \cdot \nabla \eta-\left|u\left(t_{j}\right)\right|^{p-2} u\left(t_{j}\right) \eta\right)=0 \quad \text { for all } \eta \in H_{0}^{1}(\Omega) .
$$

Using again (7.14), this tells us that (up to a subsequence)

$$
u\left(t_{j}\right) \rightarrow \phi \text { for some } \phi \in \mathscr{S} \text {. }
$$

Next, we test Eq. (1.1) with $u$ and integrate over $\left[t_{j}, t_{j}+\varepsilon_{j}\right]$ for all $j$. By using (5.6), (7.15) and arguing as above we obtain

$$
\lim _{j \rightarrow \infty} \int_{t_{j}}^{t_{j}+\varepsilon_{j}} \int_{\Omega}\left(|\nabla u(\tau)|^{2}-|u(\tau)|^{p}\right) \mathrm{d} \tau=0 .
$$

Therefore, we can find a new sequence $\tilde{t}_{j} \in\left[t_{j}, t_{j}+\varepsilon_{j}\right]$ such that

$$
\lim _{j \rightarrow \infty}\left\|\nabla u\left(\tilde{t}_{j}\right)\right\|_{2}^{2}-\left\|u\left(\tilde{t}_{j}\right)\right\|_{p}^{p}=0 .
$$

By Lemma 7.1 we may take $\tilde{t}_{j}=t_{j}$ so that Rellich Theorem yields

$$
\lim _{j \rightarrow \infty}\left\|\nabla u\left(t_{j}\right)\right\|_{2}^{2}=\lim _{j \rightarrow \infty}\left\|u\left(t_{j}\right)\right\|_{p}^{p}=\|\phi\|_{p}^{p}=\|\nabla \phi\|_{2}^{2},
$$

which, combined with (7.16), shows that $u\left(t_{j}\right) \rightarrow \phi$ strongly in $H_{0}^{1}(\Omega)$.

Concerning the proof of (3.4), since the energy functional $E$ is decreasing and bounded below, there exists $\ell \in[d, E(0))$ such that $E(t) \rightarrow \ell$ as $t \rightarrow \infty$. The fact that $\mathscr{S}_{\ell} \neq \emptyset$ and

$$
\lim _{t \rightarrow \infty} \operatorname{dist}_{H_{0}^{1}}\left(u(t), \mathscr{S}_{\ell}\right)=0
$$

follows immediately by [11, Corollaire 2.1.9] once we observe that $\mathscr{E}$ is a strict Lyapunov function for the dynamical system associated with (1.1). Notice that, as a consequence of (7.17), the stationary solution $\phi$ defined through (7.16) belongs to $\mathscr{S}_{\ell}$, being

$$
\operatorname{dist}_{H_{0}^{1}}\left(\phi, \mathscr{S}_{\ell}\right)=\lim _{j \rightarrow \infty} \operatorname{dist}_{H_{0}^{1}}\left(u\left(t_{j}\right), \mathscr{S}_{\ell}\right)=0 .
$$

Finally, again in light of (7.17), it is readily seen that

$$
\lim _{t \rightarrow \infty} J(u(t))=\ell,
$$

which, by the definition of $E(t)$, immediately yields $\left\|u_{t}(t)\right\|_{2} \rightarrow 0$.

The proof of Theorem 3.4 is now complete. 


\subsection{Proof of Theorem 3.6}

We provide a new proof of the boundedness of global solutions that parallels the one we performed in the case $\omega>0$. According to Esquivel-Avila [5, Theorems 2.8 and 3.1], the difficult part of the statement is the $L^{2}$-boundedness of the global solution.

Assume by contradiction that there exists a diverging sequence $\left\{t_{j}\right\} \subset \mathbb{R}_{+}$such that $\left\|u\left(t_{j}\right)\right\|_{2} \rightarrow \infty$. Then, by continuity, we can find a diverging sequence $\left\{\bar{t}_{m}\right\} \subset \mathbb{R}_{+}$such that $\left\|u\left(\bar{t}_{m}\right)\right\|_{2}^{2}=m^{2}+1$. Moreover, since Lemma 7.1 with $\omega=0$ reads as

$$
\lim _{\tau \rightarrow \infty} \sup \left\{\kappa>0:\|u(t)-u(t+\kappa)\|_{2}<1, \forall t \geqslant \tau\right\}=\infty,
$$

we find a second diverging sequence $\left\{\tau_{m}\right\} \subset \mathbb{R}_{+}$such that

$$
m^{2} \leqslant\|u(t)\|_{2}^{2} \leqslant m^{2}+2 \text { for every } t \in\left[\bar{t}_{m}, \bar{t}_{m}+\tau_{m}\right] .
$$

Notice that, by (7.2), as in the case $\omega>0$, inequality (7.7) holds true for some $t_{m} \in\left[\bar{t}_{m}, \bar{t}_{m}+1\right]$. By inequality (7.3) with $\omega=0$ and Poincaré inequality we deduce that

$$
\frac{\mathrm{d}}{\mathrm{d} t}\left(\int_{\Omega} u(t) u_{t}(t)+\frac{\mu}{2}\|u(t)\|_{2}^{2}\right) \geqslant \frac{p-2}{2} \lambda_{1}\|u(t)\|_{2}^{2}-p E(0) .
$$

Notice that

$$
\int_{\Omega} u\left(t_{m}\right) u_{t}\left(t_{m}\right)+\frac{\mu}{2}\left\|u\left(t_{m}\right)\right\|_{2}^{2} \geqslant 0
$$

for $m$ large enough. Indeed, by (7.7), (7.18) and by Young and Hölder inequalities,

$$
\begin{aligned}
\int_{\Omega} u\left(t_{m}\right) u_{t}\left(t_{m}\right)+\frac{\mu}{2}\left\|u\left(t_{m}\right)\right\|_{2}^{2} & \geqslant \frac{\mu}{2}\left\|u\left(t_{m}\right)\right\|_{2}^{2}-\left\|u\left(t_{m}\right)\right\|_{2}\left\|u_{t}\left(t_{m}\right)\right\|_{2} \\
& \geqslant \frac{\mu}{4}\left\|u\left(t_{m}\right)\right\|_{2}^{2}-C_{\mu}\left\|u_{t}\left(t_{m}\right)\right\|_{2}^{2} \geqslant \frac{\mu}{4} m^{2}-2 C_{\mu} d \geqslant 0
\end{aligned}
$$

for some $C_{\mu}>0$, provided that $m$ is large enough. Let us fix $m \geqslant 1$ so large that $m^{2} \geqslant(4 p /(p-2)) E(0) / \lambda_{1}$ and inequalities (7.7), (7.18) and (7.20) hold true. By (7.18) and (7.20), integrating (7.19) on the time interval $\left[t_{m}, t\right]$ for $t \in\left(t_{m}, t_{m}+\tau_{m}\right]$ entails

$$
\int_{\Omega} u(t) u_{t}(t)+\frac{\mu}{2}\|u(t)\|_{2}^{2} \geqslant \int_{t_{m}}^{t}\left(\frac{p-2}{2} \lambda_{1}\|u(\tau)\|_{2}^{2}-p E(0)\right) \mathrm{d} \tau \geqslant \frac{p-2}{4} \lambda_{1} \int_{t_{m}}^{t}\|u(\tau)\|_{2}^{2} \mathrm{~d} \tau .
$$

On the other hand, by Hölder and Young inequalities,

$$
\int_{\Omega} u(t) u_{t}(t)+\frac{\mu}{2}\|u(t)\|_{2}^{2} \leqslant \frac{1}{2}\left\|u_{t}(t)\right\|_{2}^{2}+C_{\mu}\|u(t)\|_{2}^{2}
$$

for some $C_{\mu}>0$. In conclusion, if we set

$$
\bar{\Upsilon}_{m}(t)=\int_{t_{m}}^{t}\|u(\tau)\|_{2}^{2} \mathrm{~d} \tau \quad \text { for } t \in\left(t_{m}, t_{m}+\tau_{m}\right],
$$

then the following differential inequality is satisfied

$$
\bar{\Upsilon}_{m}^{\prime}(t) \geqslant \bar{\gamma} \bar{\Upsilon}_{m}(t)-\bar{C}\left\|u_{t}(t)\right\|_{2}^{2} \text { for every } t \in\left(t_{m}, t_{m}+\tau_{m}\right]
$$


for some $\bar{\gamma}>0$ and $\bar{C}>0$. At this point, taking into account (7.18), the desired contradiction pops up exactly as in the proof of Theorem 3.4. The other assertions in the statement of Theorem 3.6 follow arguing as in the case $\omega>0$.

Remark 7.2. A general criterion to establish the precompactness of bounded trajectories of a dynamical system was issued in a celebrated paper due to Webb [30]. In fact, in the proof of Theorems 3.4 and 3.6, the strong convergence of $\left\{u\left(t_{j}\right)\right\}$ for a suitable diverging sequence $\left\{t_{j}\right\}$ could also be obtained as a byproduct of [30, Proposition 3.1] by splitting the solution semigroup $S(t)$ into the sum of an exponentially decaying linear semigroup $S_{1}(t)$ and of a completely continuous nonlinear mapping $S_{2}(t)$. On the other hand, in our proof, once the weak limit of $\left\{u\left(t_{j}\right)\right\}$ is identified as a stationary solution $\phi \in \mathscr{S}$, taking into account the crucial stabilization property given by Lemma 7.1, the strong convergence is recovered at once by a simple variational argument.

\section{Proof of Theorem 3.12}

We start with the following elementary statement.

Lemma 8.1. Let $\delta \geqslant 0, T>0$ and let $h$ be a Lipschitzian function over $[0, T)$. Assume that $h(0) \geqslant 0$ and $h^{\prime}(t)+$ $\delta h(t)>0$ for a.e. $t \in\left(0, T_{\max }\right)$. Then $h(t)>0$ for all $t \in(0, T)$.

If $u$ solves (1.1), by [24, Lemma 4.1] we know that the map $\left\{t \mapsto \frac{\mathrm{d}^{2}}{\mathrm{~d} t^{2}}\|u(t)\|_{2}^{2}\right\}$ is defined for a.e. $t$. Hence, if $\omega=0$ and $\mu \geqslant 0$, the identity (5.7) reads as

$$
\frac{\mathrm{d}^{2}}{\mathrm{~d} t^{2}}\|u(t)\|_{2}^{2}+\mu \frac{\mathrm{d}}{\mathrm{d} t}\|u(t)\|_{2}^{2}=2\left[\left\|u_{t}(t)\right\|_{2}^{2}-I(u(t))\right] \quad \text { for a.e. } t \in\left[0, T_{\max }\right) .
$$

We may now prove the weak antidissipativity of the flow whenever $u(t) \in \mathscr{N}_{-}$.

Lemma 8.2. Assume that (1.4) hold and that $\omega=0$ and $\mu \geqslant 0$. In addition, assume that $u_{0} \in \mathscr{N}_{-}$and $u_{1} \in L^{2}(\Omega)$ are such that

$$
\int_{\Omega} u_{0} u_{1} \geqslant 0 .
$$

Let $u$ be the solution of (1.1) with initial data $\left(u_{0}, u_{1}\right)$. Then the map $\left\{t \mapsto\|u(t)\|_{2}\right\}$ is strictly increasing as long as $u(t) \in \mathscr{N}_{-}$.

Proof. Let $F(t)=\|u(t)\|_{2}^{2}$ and $G(t)=F^{\prime}(t)=2 \int_{\Omega} u u_{t}$. By [24, Lemma 4.1], the function $G$ is Lipschitzian. Note also that $G(0)=2 \int_{\Omega} u_{0} u_{1} \geqslant 0$ and that $G$ satisfies

$$
G^{\prime}(t)+\mu G(t)>0 \text { for a.e. } t \in\left[0, T_{\max }\right)
$$

in view of $(8.1)$ and of the fact that $u(t) \in \mathscr{N}_{-}$(so that $I(u(t))<0$ ). Therefore, Lemma 8.1 applies and hence $F$ is strictly increasing as long as $u(t) \in \mathscr{N}_{-}$.

In order to prove Theorem 3.12, we first claim that the solution $u$ satisfies

$$
u(t) \in \mathscr{N}_{-} \quad \text { for every } t \in\left[0, T_{\max }\right) .
$$

If this was not the case, then there would exist a first time $T \in\left(0, T_{\max }\right)$ where $u(t)$ exits $\mathscr{N}_{-}$, that is, $u(T) \in \mathscr{N}$. By Lemma 8.2, we infer that

$$
\|u(T)\|_{2}>\left\|u_{0}\right\|_{2} \geqslant \Lambda_{E(0)} .
$$


Moreover, by (4.13) (notice that $E$ is constant if $\omega=\mu=0$ ) we get

$$
J(u(T)) \leqslant E(T) \leqslant E(0) .
$$

This shows that $u(T) \in \mathscr{N} \cap J^{E(0)}=\mathscr{N}_{E(0)}$. Together with (8.3) and the definition of $\Lambda_{E(0)}$, this leads to a contradiction and proves (8.2).

By contradiction, assume now that $u$ is global, namely $T_{\max }=\infty$. Then, by energy arguments analogous to those in the proof of Theorem 3.4, there exist $\phi \in \mathscr{N}_{E(0)}$ and a diverging sequence $\left\{t_{j}\right\}$ such that $u\left(t_{j}\right) \rightarrow \phi$ in $H_{0}^{1}(\Omega)$, so that $\|\phi\|_{2} \leqslant \Lambda_{E(0)}$. But Lemma 8.2 and (8.2) give

$$
\|\phi\|_{2}>\left\|u_{0}\right\|_{2} \geqslant \Lambda_{E(0)},
$$

a contradiction. Theorem 3.12 is so proved.

\section{Proof of Theorem 3.13}

We first recall a simple property of $\mathscr{N}$ (see also [8, Theorem 15]).

Lemma 9.1. Let $\beta$ be as in (2.4). Then, for any $\sigma \geqslant \beta$ and for any $k \geqslant 1$ there exists $u \in \mathscr{N}$ such that $\operatorname{supp}(u)=$ $\overline{\Omega / k}$ and $\|\nabla u\|_{2}=\sigma$, where $\operatorname{supp}(u)$ is the support of $u$.

Proof. Since $\mathscr{N}$ is unbounded and connected, for $k=1$ and any $\sigma \geqslant \beta$ there exists a function $u$ with the required properties. In the case $k>1$ it is sufficient to rescale $u$ as

$$
u_{k}(x)= \begin{cases}k^{2 /(p-1)} u(k x) & \text { for } x \in \frac{\operatorname{supp}(u)}{k}, \\ 0 & \text { for } x \notin \frac{\operatorname{supp}(u)}{k},\end{cases}
$$

to get a function $u \in \mathscr{N}$.

We will construct $\left(u_{0}^{m}, u_{1}^{m}\right)$ as in the statement of Theorem 3.13 by using Theorem 3.12. Fix $m>0$ sufficiently large and take $u_{1}^{m} \equiv 0$. Take any $v \in H_{0}^{1}(\Omega) \backslash\{0\}$ such that $\operatorname{supp}(v) \subset\left(\Omega \backslash \frac{\Omega}{2}\right)$. Then, take $\alpha_{m}>0$ sufficiently large so that

$$
\alpha_{m}\|v\|_{2} \geqslant \Lambda_{m}, \quad J\left(\alpha_{m} v\right)<0 .
$$

By Lemma 9.1, we may find $w_{m} \in \mathscr{N} \operatorname{such}$ that $\operatorname{supp}\left(w_{m}\right) \subset \overline{\Omega / 2}$ and

$$
J\left(w_{m}\right)=\frac{p-2}{2 p}\left\|\nabla w_{m}\right\|_{2}^{2}=m-J\left(\alpha_{m} v\right)>m .
$$

Finally, let $u_{0}^{m}=w_{m}+\alpha_{m} v$. Then, since $w_{m}$ and $v$ have disjoint supports, by (9.1) and (9.2) we have

$$
\begin{aligned}
& \left\|u_{0}^{m}\right\|_{2}=\left\|w_{m}\right\|_{2}+\alpha_{m}\|v\|_{2}>\Lambda_{m}, \\
& J\left(u_{0}^{m}\right)=J\left(w_{m}\right)+J\left(\alpha_{m} v\right)=m, \\
& I\left(u_{0}^{m}\right)=I\left(w_{m}\right)+I\left(\alpha_{m} v\right)=I\left(\alpha_{m} v\right)<0,
\end{aligned}
$$

the latter inequality following from (9.1) and the fact that $J$ is nonnegative in $\mathscr{N}_{+} \cup \mathscr{N}$. Moreover, $\mathscr{E}\left(u_{0}^{m}, u_{1}^{m}\right)=$ $J\left(u_{0}^{m}\right)=m$. Hence, $\left(u_{0}^{m}, u_{1}^{m}\right)$ satisfies all the assumptions of Theorem 3.12 and the proof of Theorem 3.13 is complete. 


\section{References}

[1] A. Ambrosetti, P.H. Rabinowitz, Dual variational methods in critical point theory and applications, J. Funct. Anal. 14 (1973) $349-381$.

[2] J. Ball, Global attractors for damped semilinear wave equations, Discrete Contin. Dyn. Syst. 10 (2004) 31-52.

[3] A.N. Carvalho, J.W. Cholewa, Local well posedness for strongly damped wave equations with critical nonlinearities, Bull. Austral. Math. Soc. 66 (2002) 443-463.

[4] T. Cazenave, Uniform estimates for solutions of nonlinear Klein-Gordon equations, J. Funct. Anal. 60 (1985) 36-55.

[5] J. Esquivel-Avila, The dynamics of a nonlinear wave equation, J. Math. Anal. Appl. 279 (2003) 135-150.

[6] J. Esquivel-Avila, Qualitative analysis of a nonlinear wave equation, Discrete Contin. Dyn. Syst. 10 (2004) $787-804$.

[7] F. Gazzola, Finite time blow-up and global solutions for some nonlinear parabolic equations, Differential Integral Equations 17 (2004) 983-1012.

[8] F. Gazzola, T. Weth, Finite time blow-up and global solutions for semilinear parabolic equations with initial data at high energy level, Differential Integral Equations, in press.

[9] V. Georgiev, G. Todorova, Existence of a solution of the wave equation with nonlinear damping and source term, J. Differential Equations 109 (1994) 295-308.

[10] J.K. Hale, G. Raugel, Convergence in gradient-like systems with applications to PDE, Z. Angew. Math. Phys. 43 (1992) $63-124$.

[11] A. Haraux, Dissipative Dynamical Systems and Applications, Res. Appl. Math., vol. 17, Masson, Paris, 1991, 132 p.

[12] A. Haraux, M.A. Jendoubi, Convergence of bounded weak solutions of the wave equation with dissipation and analytic nonlinearity, Calc. Var. Partial Differential Equations 9 (1999) 95-124.

[13] R. Ikehata, Some remarks on the wave equations with nonlinear damping and source terms, Nonlinear Anal. 27 (1996) $1165-1175$.

[14] R. Ikehata, T. Suzuki, Stable and unstable sets for evolution equations of parabolic and hyperbolic type, Hiroshima Math. J. 26 (1996) 475-491.

[15] M.A. Jendoubi, P. Poláčik, Non-stabilizing solutions of semilinear hyperbolic and elliptic equations with damping, Proc. Roy. Soc. Edinburgh Sect. A 133 (2003) 1137-1153.

[16] H.A. Levine, Instability and nonexistence of global solutions to nonlinear wave equations of the form $P u_{t t}=-A u+\mathcal{F}(u)$, Trans. Amer. Math. Soc. 192 (1974) 1-21.

[17] H.A. Levine, Some additional remarks on the nonexistence of global solutions to nonlinear wave equations, SIAM J. Math. Anal. 5 (1974) 138-146.

[18] H.A. Levine, J. Serrin, Global nonexistence theorems for quasilinear evolution equations with dissipation, Arch. Rational Mech. Anal. 137 (1997) 341-361.

[19] H.A. Levine, G. Todorova, Blow up of solutions of the Cauchy problem for a wave equation with nonlinear damping and source terms and positive initial energy, Proc. Amer. Math. Soc. 129 (2001) 793-805.

[20] Z. Nehari, On a class of nonlinear second-order differential equations, Trans. Amer. Math. Soc. 95 (1960) 101-123.

[21] M. Ohta, Remarks on blowup of solutions for nonlinear evolution equations of second order, Adv. Math. Sci. Appl. 8 (1998) 901-910.

[22] K. Ono, On global existence, asymptotic stability and blowing up of solutions for some degenerate non-linear wave equations of Kirchhoff type with a strong dissipation, Math. Methods Appl. Sci. 20 (1997) 151-177.

[23] V. Pata, M. Squassina, On the strongly damped wave equation, Comm. Math. Phys. 253 (2004) 511-533.

[24] L.E. Payne, D.H. Sattinger, Saddle points and instability of nonlinear hyperbolic equations, Israel Math. J. 22 (1975) $273-303$.

[25] P. Pucci, J. Serrin, Global nonexistence for abstract evolution equations with positive initial energy, J. Differential Equations 150 (1998) 203-214.

[26] P. Pucci, J. Serrin, Some new results on global nonexistence for abstract evolution with positive initial energy, Topol. Methods Nonlinear Anal. 10 (1997) 241-247.

[27] D.H. Sattinger, On global solution of nonlinear hyperbolic equations, Arch. Rational Mech. Anal. 30 (1968) 148-172.

[28] M. Tsutsumi, On solutions of semilinear differential equations in a Hilbert space, Math. Japon. 17 (1972) 173-193.

[29] E. Vitillaro, Global existence theorems for a class of evolution equations with dissipation, Arch. Rational Mech. Anal. 149 (1999) $155-182$.

[30] G.F. Webb, Compactness of bounded trajectories of dynamical systems in infinite-dimensional spaces, Proc. Roy. Soc. Edinburgh Sect. A 84 (1979) 19-33.

[31] M. Willem, Minimax Theorems, Progress Nonlinear Differential Equations Appl., vol. 24, Birkhäuser Boston, Boston, MA, 1996,162 p. 